\title{
Modeling and Control for Max-Plus Systems with Partial Synchronization
}

\author{
X. David-Henriet ${ }^{*, * *, * * *}$ J. Raisch ${ }^{*, * *}$ L. Hardouin ${ }^{* *}$ \\ B. Cottenceau ${ }^{* *}$ \\ * Technische Universität Berlin, Fachgebiet Regelungssysteme, \\ 10587 Berlin, Germany (e-mail: david-henriet@control.tu-berlin.de) \\ ** Laboratoire d'Ingénierie des Systèmes Automatisés, LARIS, ISTIA, \\ Université d'Angers, Angers 49000, France \\ (e-mail: laurent.hardouin@univ-angers.fr) \\ *** Max-Planck-Institut für Dynamik komplexer technischer Systeme, \\ Fachgruppe System- und Regelungstheorie, 39106 Magdeburg, \\ Germany
}

\begin{abstract}
A max-plus system with partial synchronization is a persistent discrete event system divided into a main subsystem and a secondary subsystem where some events in the secondary subsystem can only occur when (not after) associated events in the main subsystem occur. A formal model based on max-plus recursive equations is presented for max-plus systems with partial synchronization. Furthermore, the control problem of output tracking is addressed by assigning a higher priority to the main subsystem: the performance of the main subsystem is never degraded to improve the performance of the secondary subsystem. Two different control approaches are investigated: optimal feedforward control and model predictive control. In both cases, residuation theory is applied to efficiently solve the optimization problem.
\end{abstract}

Keywords: Discrete event systems, max-plus systems, synchronization, modeling, optimal control, model predictive control.

\section{INTRODUCTION}

A discrete event system is said to be persistent if the occurrence of an event never disables another event. Then, in a persistent discrete event system, an enabled event remains enabled until it occurs. Such systems may appear after solving the conflicts between events by a predefined schedule in the fields of manufacturing processes or transportation networks. The times when events occur often play an important role in the dynamics of persistent discrete event systems. Therefore, the fundamental question is not about the occurrence of an enabled event, but about its occurring time. In many applications, the time behavior of a persistent discrete event system is described by synchronization rules of the following form: occurrence $k$ of event $i$ occurs at least $\tau$ units of time after occurrence $k-l$ of event $j$. This kind of synchronization is referred to in the following as standard synchronization and leads to recursive equations for the occurring times of events. After rewriting these equations in the max-plus algebra, linear equations similar to state-space equations in classical control theory appear. Therefore, such systems are called max-plus linear systems. By analogy with classical control theory, transfer functions are defined for max-plus linear systems using operatorial representation (Cohen et al. (1989) and Baccelli et al. (1992)). Moreover, several methods from classical control theory have been adapted to max-plus linear systems, e.g., optimal feedforward control (Cohen et al. (1993) and Lhommeau et al. (2003)), model predictive control (De Schutter and van den Boom (2001)), and feedback control (Cottenceau et al. (2001)).

In the literature, synchronization rules different from standard synchronization have already been investigated. Soft synchronization, introduced in De Schutter and van den Boom (2003), is defined as a standard synchronization which can be ignored at a certain cost. Exact synchronization of event $i$ and event $j$ corresponds to the following rule: occurrence $k$ of event $i$ and of event $j$ occur simultaneously. Obviously, exact synchronization comes down to two standard synchronizations: occurrence $k$ of event $i$ occurs after occurrence $k$ of event $j$, and, conversely, occurrence $k$ of event $j$ occurs after occurrence $k$ of event $i$. Partial synchronization is another synchronization rule introduced in David-Henriet et al. (2013) corresponding to an asymmetric form of exact synchronization. The partial synchronization of event $i$ by event $j$ means that event $i$ can only occur when (not after) event $j$ occurs.

In this paper, a particular class of persistent discrete event systems is considered. The time behavior of these systems is ruled only by standard synchronizations and partial synchronizations. The system is divided into two disjoint (i.e., with disjoint event sets) subsystems (a main subsystem and a secondary subsystem) such that only standard synchronizations of events in the same subsystem and partial synchronizations of events in the secondary subsystem by events in the main subsystem exist. Such systems are called max-plus systems with partial synchronization. In public transportation networks, for example, 
a user (secondary subsystem) waiting for a bus (main subsystem) can only continue his travel when he gets in a bus, however a bus does not need to wait at a bus stop for users in order to continue its travel. Other applications in the field of transportation network are, for example, transportation networks with traffic lights (e.g., a car can pass a traffic light when the traffic light is green) and hierarchical public transportation networks (e.g., only connections with an upper level are ensured). In David-Henriet et al. (2013), this class of systems has been investigated under a predefined behavior of the main subsystem and, under this assumption, optimal feedforward control for the secondary subsystem has been defined. In this paper, this restriction is dropped: the main subsystem is a maxplus linear system with its own dynamics. Furthermore, optimal feedforward control and model predictive control (MPC) are investigated for max-plus systems with partial synchronization.

Necessary mathematical tools are recalled in $\S 2$. In $\S 3$, a model for max-plus systems with partial synchronization is presented. The main contributions of this paper are introduced in $\S 4$ : optimal feedforward control and MPC for max-plus systems with partial synchronization.

\section{MATHEMATICAL PRELIMINARIES}

The following is a short summary of basic results from dioid theory and residuation theory. The reader is invited to consult Cohen et al. (1989) and Baccelli et al. (1992) for more details.

\subsection{Dioid Theory}

A dioid $\mathcal{D}$ is a set endowed with two internal operations $\oplus$ (addition) and $\otimes$ (multiplication, often denoted by juxtaposition), both associative and both having a neutral element denoted $\varepsilon$ and $e$ respectively. Moreover, $\oplus$ is commutative and idempotent $(\forall a \in \mathcal{D}, a \oplus a=a), \otimes$ is distributive with respect to $\oplus$, and $\varepsilon$ is absorbing for $\otimes$ $(\forall a \in \mathcal{D}, \varepsilon \otimes a=a \otimes \varepsilon=\varepsilon)$. The operation $\oplus$ induces an order relation $\preceq$ on $\mathcal{D}$, defined by $\forall a, b \in \mathcal{D}, a \preceq b \Leftrightarrow a \oplus$ $b=b$.

A dioid $\mathcal{D}$ is said to be complete if it is closed for infinite sums and if multiplication distributes over infinite sums. A complete dioid admits a greatest element $\top=\bigoplus_{x \in \mathcal{D}} x$. On a complete dioid, a new internal operation $\wedge$ is defined by, for all $a, b \in \mathcal{D}$,

$$
a \wedge b=\bigoplus_{x \in \mathcal{X}_{a, b}} x \text { with } \mathcal{X}_{a, b}=\{x \in \mathcal{D} \mid x \preceq a \text { and } x \preceq b\}
$$

Clearly, $\wedge$ is idempotent and admits $\top$ as neutral element. Endowed with $\wedge$ and $\oplus$, a complete dioid is a complete lattice (see Blyth (2005) for a survey on lattice theory).

Example 1. A well-known complete dioid is the (max, +)algebra, denoted $\overline{\mathbb{N}}_{\text {max }}: \mathbb{N}_{0} \cup\{-\infty,+\infty\}$ is endowed with max as addition and + as multiplication. $\varepsilon$ is equal to $-\infty$ and $\top$ to $+\infty$. The associated order relation $\preceq$ is the usual order relation $\leq$.

By analogy with standard linear algebra, $\oplus$ and $\otimes$ are defined for matrices with entries in a dioid. For $A, B \in$ $\mathcal{D}^{n \times m}$ and $C \in \mathcal{D}^{m \times p}$,
- $(A \oplus B)_{i j}=A_{i j} \oplus B_{i j}$

- $(A \otimes C)_{i j}=\bigoplus_{k=1}^{m} A_{i k} C_{k j}$

Furthermore, endowed with these operations, the set of square matrices with entries in a complete dioid is also a complete dioid.

\section{Daters}

Definition 2. (Dater). A dater $f$ is an isotone (i.e., orderpreserving) mapping from $\mathbb{Z}$ to the complete dioid $\overline{\mathbb{N}}_{\max }$ such that $f(k)=\varepsilon$ for $k<0$.

The set of daters, denoted $\Sigma$, inherits the operation $\oplus$ defined on $\overline{\mathbb{N}}_{\max }$. For $f_{1}, f_{2} \in \Sigma$,

$$
\forall k \in \mathbb{Z}, \quad\left(f_{1} \oplus f_{2}\right)(k)=f_{1}(k) \oplus f_{2}(k)
$$

The operation $\oplus$ admits a neutral element $\varepsilon$ in $\Sigma$ defined by, for all $k \in \mathbb{N}_{0}, \varepsilon(k)=\varepsilon$. The order relation $\preceq$ on $\overline{\mathbb{N}}_{\max }$ induces an order relation $\preceq$ on $\Sigma$. For $f_{1}, f_{2} \in \bar{\Sigma}$,

$$
f_{1} \preceq f_{2} \Leftrightarrow\left(\forall k \in \mathbb{Z}, f_{1}(k) \preceq f_{2}(k)\right)
$$

Then, endowed with $\oplus, \Sigma$ is a join semilattice.

The image of a dater, denoted $\operatorname{Im}(d)$, is defined by

$$
\operatorname{Im}(d)=\{d(k) \mid k \in \mathbb{Z}\}
$$

A dater over a finite horizon is a dater $f$ admitting $L \in \mathbb{N}_{0}$ such that, for all $k \geq L, f(k)$ is either equal to $\varepsilon$ or to $\top$ and $f(k)=f(L)$. The set of daters over a finite horizon is denoted $\Sigma_{f}$. Obviously, for all $f_{1}, f_{2} \in \Sigma_{f}, f_{1} \oplus f_{2}$ belongs to $\Sigma_{f}$.

\subsection{Residuation Theory}

In the following, some concepts and results of residuation theory are recalled. A survey is available in Blyth (2005). Definition 3. (Residuation). Let $f: E \rightarrow F$ with $E$ and $F$ ordered sets. $f$ is said to be residuated if $f$ is isotone and if, for all $y \in F$, the least upper bound of the subset $\{x \in E \mid f(x) \preceq y\}$ exists and lies in this subset. This element in $E$ is denoted $f^{\sharp}(y)$. Mapping $f^{\sharp}$ from $F$ to $E$ is called the residual of $f$.

Over complete dioids, the mapping $L_{a}: x \mapsto a \otimes x$ (leftproduct by $a$ ), respectively $R_{a}: x \mapsto x \otimes a$ (right-product by $a$ ), is residuated. Its residual is denoted by $L_{a}^{\sharp}(x)=a ф x$ (left-division by $a$ ), resp. $R_{a}^{\sharp}(x)=x \phi a$ (right-division by a). As left- and right-products are extended to matrices with entries in a complete dioid, left- and right-divisions are also extended to matrices with entries in a complete dioid.

\section{MODEL OF MAX-PLUS SYSTEMS WITH PARTIAL SYNCHRONIZATION}

In the following, a formal description of max-plus systems with partial synchronization based on daters is proposed. An event $v$ is equipped with an eponym dater $v$ such that, for $k \in \mathbb{N}_{0}, v(k)$ corresponds to the time of occurrence $k$ of event $v$.

The event set of the system is partitioned into input events $u$, state events $x$, and output events $y$ such that input events are not subject to standard synchronizations and there is no standard synchronization by output events. 
From now on, the set of input events, state events, and output events in the main subsystem and in the secondary subsystem are assumed non-empty. For many practical applications, this assumption holds. Furthermore, the system operates under the earliest functioning rule: each state or output event occurs as soon as it is enabled.

The main subsystem is a max-plus linear system, as the only synchronizations influencing its dynamics are standard synchronizations. Then, the dynamics of the main subsystem is described by

$$
\left\{\begin{array}{l}
x_{1}(k)=A_{1} x_{1}(k-1) \oplus B_{1} u_{1}(k) \\
y_{1}(k)=C_{1} x_{1}(k)
\end{array}\right.
$$

where $u_{1}, x_{1}$, and $y_{1}$ are the input, state, and output associated with the main subsystem. Matrices $A_{1} \in \overline{\mathbb{N}}_{\max }^{n_{1} \times n_{1}}$, $B_{1} \in \overline{\mathbb{N}}_{\max }^{n_{1} \times m_{1}}$, and $C_{1} \in \overline{\mathbb{N}}_{\max }^{p_{1} \times n_{1}}$ are obtained from the standard synchronizations in the main subsystem.

The equations for the dynamics of the secondary subsystem are slightly different from (1) due to partial synchronizations of events in the secondary subsystem by events in the main subsystem. Without loss of generality, it is possible to assume that there exist only partial synchronizations of state events in the secondary subsystem by state events in the main subsystem. Then, the dynamics of the secondary subsystem is given by the least solution of

$$
\left\{\begin{array}{l}
x_{2}(k) \succeq A_{2,0} x_{2}(k) \oplus A_{2,1} x_{2}(k-1) \oplus B_{2} u_{2}(k) \\
y_{2}(k)=C_{2} x_{2}(k) \\
\operatorname{Im}\left(\left(x_{2}\right)_{j}\right) \subseteq \Phi_{j} \text { for all } \mathrm{j}
\end{array}\right.
$$

where $u_{2}, x_{2}$, and $y_{2}$ are the input, state, and output associated with the secondary subsystem. Matrices $A_{2,0}, A_{2,1} \in$ $\overline{\mathbb{N}}_{\text {max }}^{n_{2} \times n_{2}}, B_{2} \in \overline{\mathbb{N}}_{\text {max }}^{n_{2} \times m_{2}}$, and $C_{2} \in \overline{\mathbb{N}}_{\text {max }}^{p_{2} \times n_{2}}$ are obtained from the standard synchronizations in the secondary subsystem. The last condition in (2) is due to partial synchronizations. $\Phi_{j}$ denotes the set of admissible (with respect to partial synchronizations) occurring times of state events $\left(x_{2}\right)_{j}$. Then, $\Phi_{j}$ is defined by

$$
\Phi_{j}=\overline{\mathbb{N}}_{\max } \cap \bigcap_{i \in S_{j}}\left(\operatorname{Im}\left(\left(x_{1}\right)_{i}\right) \cup\{\top\}\right)
$$

where $S_{j}$ is the set of indices $i$ such that there exists a partial synchronization of event $\left(x_{2}\right)_{j}$ by event $\left(x_{1}\right)_{i}$. $\top$ is always included in $\Phi_{j}$ to model the non-occurrence of event $\left(x_{2}\right)_{j}:\left(x_{2}\right)_{j}(k)=\top$ means that occurrence $k$ of event $\left(x_{2}\right)_{j}$ never happens.

A max-plus system with partial synchronization is composed of $m=m_{1}+m_{2}$ input events, $n=n_{1}+n_{2}$ state events, and $p=p_{1}+p_{2}$ output events.

\subsection{System Evolution on Finite Horizon}

In the following, a method is presented to calculate the response of a max-plus system with partial synchronization to an input $u$ divided into input $u_{1}$ corresponding to the main subsystem and input $u_{2}$ corresponding to the secondary subsystem. It is assumed that input $u$ is composed of daters over a finite horizon (i.e., $u \in \Sigma_{f}^{m}$ ): there exists $L$ in $\mathbb{N}_{0}$ such that, for $k \geq L$, all entries of $u(k)$ are equal either to $\varepsilon$ or to $\top$ and $u(k)=u(L)$. Then, an input event $u_{j}$ is either insignificant (i.e., for all $k \in \mathbb{Z}, u_{j}(k)=\varepsilon$ ) or admits at most $L$ occurrences. By injecting $u_{1}$ in (1), it appears that $x_{1}(k)=x_{1}\left(L+n_{1}\right)$ for $k \geq L+n_{1}$. Then, the time behavior of the main subsystem is completely defined after a finite number of operations. For the secondary subsystem, the sets $\Phi_{j}$ are determined by the dynamics of the main subsystem. The cardinality of $\Phi_{j}$ is bounded by $L$. The response of the secondary subsystem to $u_{2}$ is obtained using the method presented in David-Henriet et al. (2013). In the same way, the time behavior of the secondary subsystem is completely defined after a finite number of operations, as $x_{2}(k)=x_{2}\left(L+n_{2}\right)$ for $k \geq L+n_{2}$. To calculate the complete time behavior of event $\left(x_{2}\right)_{j}$, it is only necessary to scan once $\Phi_{j}$. The amount of calculations necessary to determine the response to input $u$ in $\Sigma_{f}^{m}$ is linear in $L$. To summarize, the calculation of state $x$ boils down to evaluate successively two recurrence relations over $L+\max \left(n_{1}, n_{2}\right)$ steps.

As all entries of $u(L)$ are equal either to $\varepsilon$ or to $T$, all entries of $x\left(L+\max \left(n_{1}, n_{2}\right)\right)$ and $y\left(L+\max \left(n_{1}, n_{2}\right)\right)$ are equal either to $\varepsilon$ or to $T$. Then, each state or output event is either insignificant or admits at most $L+\max \left(n_{1}, n_{2}\right)$ occurrences. Consequently, an input-output mapping $H$ from $\Sigma_{f}^{m}$ to $\Sigma_{f}^{p}$ is associated with a max-plus system with partial synchronization. $H$ is divided into input-output mappings $H_{1}$ associated with the main subsystem and $H_{2}$ associated with the secondary subsystem. $H_{1}$ is defined from $\Sigma_{f}^{m_{1}}$ to $\Sigma_{f}^{p_{1}}$. However, due to partial synchronizations of events in the secondary subsystem by events in the main subsystem, $H_{2}$ takes into account input $u_{1}$. Then, $H_{2}$ is defined from $\Sigma_{f}^{m}$ to $\Sigma_{f}^{p_{2}}$.

Remark 4. $H_{1}$ corresponds to the input-output mapping of a max-plus linear system. Therefore, using operatorial representation, $H_{1}$ has an explicit form, which allows to efficiently calculate the response of the main subsystem to periodic inputs (see Cohen et al. (1989)). However, to preserve the analogy between the main subsystem and the secondary subsystem and to simplify the discussion on complexity, it is still assumed that $u_{1}$ belongs to $\Sigma_{f}^{m_{1}}$ in the following.

The next example shows that the input-output mapping $H$ might not be isotone.

Example 5. A simple max-plus system with partial synchronization is considered. The main subsystem is described by

$$
\left\{\begin{array}{l}
x_{1}(k)=\left(\begin{array}{ll}
e & \varepsilon \\
2 & e \\
5 & 2
\end{array}\right) u_{1}(k) \\
y_{1}(k)=\left(\begin{array}{lll}
\varepsilon & \varepsilon & e
\end{array}\right) x_{1}(k)
\end{array}\right.
$$

The secondary subsystem is described by

$$
\left\{\begin{array}{l}
x_{2}(k) \succeq u_{2}(k) \\
y_{2}(k)=x_{2}(k) \\
\operatorname{Im}\left(\left(x_{2}\right)_{1}\right) \subseteq \Phi_{1}
\end{array}\right.
$$

where $x_{2}$ is partially synchronized by $\left(x_{1}\right)_{2}$. First, we consider input $u^{\prime}$ defined by

$$
\begin{aligned}
& \left(u_{1}^{\prime}\right)_{1}(k)=\left(u_{1}^{\prime}\right)_{2}(k)=\left\{\begin{array}{l}
\varepsilon \text { if } k<0 \\
e \text { if } k=0 \\
\top \text { if } k>0
\end{array}\right. \\
& u_{2}^{\prime}(k)=\left\{\begin{array}{l}
\varepsilon \text { if } k<0 \\
3 \text { if } k=0 \\
\top \text { if } k>0
\end{array}\right.
\end{aligned}
$$


This leads to output $y^{\prime}$ with

$$
y_{1}^{\prime}(k)=\left\{\begin{array}{l}
\varepsilon \text { if } k<0 \\
5 \text { if } k=0 \\
\top \text { if } k>0
\end{array} \text { and } y_{2}^{\prime}(k)=\left\{\begin{array}{l}
\varepsilon \text { if } k<0 \\
\top \text { if } k \geq 0
\end{array}\right.\right.
$$

Second, we consider the input $u^{\prime \prime}$ defined by $\left(u_{1}^{\prime \prime}\right)_{1}=\left(u_{1}^{\prime}\right)_{1}$, $u_{2}^{\prime \prime}=u_{2}^{\prime}$, and

$$
\left(u_{1}^{\prime \prime}\right)_{2}(k)=\left\{\begin{array}{l}
\varepsilon \text { if } k<0 \\
3 \text { if } k=0 \\
\top \text { if } k>0
\end{array}\right.
$$

This leads to output $y^{\prime \prime}$ with $y_{1}^{\prime \prime}=y_{1}^{\prime}$ and

$$
y_{2}^{\prime \prime}(k)=\left\{\begin{array}{l}
\varepsilon \text { if } k<0 \\
3 \text { if } k=0 \\
\top \text { if } k>0
\end{array}\right.
$$

Therefore, $u^{\prime} \prec u^{\prime \prime}$ and $y^{\prime \prime} \prec y^{\prime}$. The input-output mapping of the considered max-plus system with partial synchronization is not isotone.

\section{CONTROL OF MAX-PLUS SYSTEMS WITH PARTIAL SYNCHRONIZATION}

The control objective is to track an output reference $z$ divided into output reference $z_{1}$ corresponding to the main subsystem and output reference $z_{2}$ corresponding to the secondary subsystem. The priority is given to the performance of the main subsystem over the one of the secondary subsystem: the performance of the main subsystem shall not be degraded to improve the performance of the secondary subsystem. E.g., in a public transportation network, a bus is not delayed by the late arrival of an user. The control problem is first solved for the main subsystem by neglecting the secondary subsystem. Second, it is solved for the secondary subsystem using the predefined behavior of the main subsystem. Hence, the secondary subsystem takes into account the output reference of the main subsystem, but the main subsystem is not affected by the output reference of the secondary subsystem. Formally, this is expressed by a lexico-graphic order relation, denoted $\preceq_{\Phi}$, based on the the partition in main subsystem and secondary subsystem. For the input,

$$
u \preceq_{\Phi} u^{\prime} \Leftrightarrow\left\{\begin{array}{c}
u_{1} \prec u_{1}^{\prime} \\
\text { or } \\
u_{1}=u_{1}^{\prime} \text { and } u_{2} \preceq u_{2}^{\prime}
\end{array}\right.
$$

A similar order relation holds for the state and the output. To avoid ambiguity between the order relations $\preceq_{\Phi}$ and $\preceq$, the order relation $\preceq_{\Phi}$ is always explicitly mentioned whenever it is considered. In this section, two different control approaches are investigated: optimal feedforward control and model predictive control.

\subsection{Optimal Feedforward Control}

For optimal feedforward control, the output reference $z$ is composed of predetermined daters over a finite horizon. To simplify the following discussion, it is assumed that all entries from $z$ are different from $\varepsilon$. Then, there exists $L$ in $\mathbb{N}_{0}$ such that $z_{i}(L)=\top$ with $1 \leq i \leq p$. The problem is to find an optimal (according to a criterion given below) input, denoted $u_{o p t}$, ensuring that output events occur at the latest at the occurring times specified by the output reference $z$. Formally, this corresponds to $H\left(u_{\text {opt }}\right) \preceq z$.
This problem has already been solved for max-plus linear systems described by

$$
\left\{\begin{array}{l}
x(k)=A x(k-1) \oplus B u(k) \\
y(k)=C x(k)
\end{array}\right.
$$

with $A \in \overline{\mathbb{N}}_{\max }^{n \times n}, B \in \overline{\mathbb{N}}_{\max }^{n \times m}$, and $C \in \overline{\mathbb{N}}_{\max }^{p \times n}$. In this case, the optimal input $u_{\text {opt }}$ is defined as the greatest input $u$ such that $H(u) \preceq z$. This is known as the just-in-time criterion: the input events occur as late as possible while ensuring $H(u) \preceq z$. The reference output $z$ is tracked from below as closely as possible. The calculation of $u_{\text {opt }}$ is completed using the backward recursive equations introduced in Cohen et al. (1993),

$$
\left\{\begin{array}{l}
\zeta(k)=A \phi \zeta(k+1) \wedge C \phi z(k) \\
u_{\text {opt }}(k)=B \phi \zeta(k)
\end{array}\right.
$$

where $\zeta$ is the co-state associated with $z$ (i.e., the greatest state leading to an output less than or equal to $z$ ). As all entries of $z(L)$ are equal to $\top$, all entries of $\zeta(L)$ and $u_{\text {opt }}(L)$ are equal to $\top$. Then, $u_{\text {opt }}$ is completely defined after a finite number of operations. The input-output mapping $H$ from $\Sigma_{f}^{m}$ to $\Sigma_{f}^{p}$ associated with (3) is isotone and, for all $z \in \Sigma_{f}^{p}, H(u) \preceq z$ admits a greatest solution in $\Sigma_{f}^{m}$. Therefore, $H$ is residuated and $u_{\text {opt }}=H^{\sharp}(z)$.

For max-plus systems with partial synchronization, the optimal input is the greatest (with respect to $\preceq_{\Phi}$ ) input $u$ such that $H(u) \preceq z$. The output reference $z$ is tracked from below as closely as possible taking into account the higher priority assigned to the main subsystem.

First, the optimal input of the main subsystem, denoted $u_{1 o p t}$, is obtained by neglecting the secondary subsystem. This comes down to the calculation of the optimal input of a max-plus linear system, i.e., $u_{1 o p t}=H_{1}^{\sharp}\left(z_{1}\right)$. Second, the state of the main subsystem is obtained by injecting $u_{1 o p t}$ in (1) and yields $\Phi_{\text {opt }, j}$ with $1 \leq j \leq n_{2}$. As $u_{1 \text { opt }}$ belongs to $\Sigma_{f}^{m_{1}}$, this step terminates after a finite number of operations. Third, the optimal input of the secondary subsystem, denoted $u_{2 o p t}$, is calculated under the assumption $u_{1}=u_{1 o p t}$. Then, $u_{2 o p t}$ is the greatest solution of

$$
\left\{\begin{array}{l}
\zeta_{2}(k) \preceq A_{2,0} \phi \zeta_{2}(k) \wedge A_{2,1} \phi \zeta_{2}(k+1) \wedge C_{2} \phi z_{2}(k) \\
u_{2 o p t}(k)=B_{2} \phi \zeta_{2}(k) \\
\operatorname{Im}\left(\left(\zeta_{2}\right)_{j}\right) \subseteq \Phi_{\text {opt }, j} \text { with } 1 \leq j \leq n_{2}
\end{array}\right.
$$

where $\zeta_{2}$ is the co-state of the secondary subsystem associated with $z_{2}$ (i.e., the greatest state leading to an output less than or equal to $z_{2}$ ). As all entries of $z_{2}(L)$ are equal to $\top$, all entries of $\zeta_{2}(L)$ and $u_{2 o p t}(L)$ are equal to $\top$. Then, $u_{2 o p t}$ is obtained after a finite number of operations using the approach developed in David-Henriet et al. (2013). The mapping $H_{2, z_{1}}$ defined by

$$
H_{2, z_{1}}\left(u_{2}\right)=H_{2}\left(H_{1}^{\sharp}\left(z_{1}\right), u_{2}\right)
$$

is an input-output mapping for the secondary system parametrized by the output reference $z_{1}$ of the main subsystem. This mapping is isotone and, for all $z_{2} \in$ $\Sigma_{f}^{p_{2}}, H_{2, z_{1}}(u) \preceq z_{2}$ admits a greatest solution in $\Sigma_{f}^{m_{2}}$. Therefore, $H_{2, z_{1}}$ is residuated and $u_{2 o p t}=H_{2, z_{1}}^{\sharp}\left(z_{2}\right)$. By analogy with the response to an input, the amount of calculations necessary to calculate the optimal input associated with an output reference $z$ in $\Sigma_{f}^{p}$ is linear in $L$. 
Remark 6. The input-output mapping $H$ from $\Sigma_{f}^{m}$, endowed with $\preceq_{\Phi}$, to $\Sigma_{f}^{p}$ might not be isotone: in Ex. 5 , $u^{\prime} \prec_{\Phi} u^{\prime \prime}$ and $y^{\prime \prime} \prec y^{\prime}$. Then, $H$ might not be residuated. However, for all $z$ in $\Sigma_{f}^{p}$, the least upper bound (with respect to $\left.\preceq_{\Phi}\right)$ of the subset $\left\{u \in \Sigma_{f}^{m} \mid H(u) \preceq z\right\}$ exists and lies in this subset.

Feasible Reference Output The system might not be able to respect the output reference $z$. This translates into an unrealizable optimal input: some entries of $u_{\text {opt }}(0)$ are equal to $\varepsilon$. We then consider the problem of finding the least (with respect to $\preceq_{\Phi}$ ) feasible output reference $z^{\prime}$ greater than or equal to $z$. Optimality of $z^{\prime}$ with respect to $\preceq_{\Phi}$ ensures that the performance of the main subsystem is not degraded to improve the performance of the secondary subsystem. The first step focuses on the main subsystem and neglects the secondary subsystem. The aim is to determine the least feasible output reference of the main subsystem $z_{1}^{\prime}$ greater than or equal to $z_{1}$. An unrealizable input such that $H_{1}\left(u_{1}\right) \preceq z_{1}$ is $\varepsilon_{1 L}$, the entries of which are all equal to $\varepsilon_{L}$ defined by

$$
\varepsilon_{L}(k)=\left\{\begin{array}{l}
\varepsilon \text { if } k<L \\
\top \text { if } k \geq L
\end{array}\right.
$$

Then, $H_{1}^{\sharp}\left(z_{1}^{\prime}\right) \succeq H_{1}^{\sharp}\left(z_{1}\right) \succeq \varepsilon_{1 L}$. The least realizable input greater than or equal to $\varepsilon_{1 L}$ is $e_{1 L}$, the entries of which are all equal to $e_{L}$ defined by

$$
e_{L}(k)=\left\{\begin{array}{l}
\varepsilon \text { if } k<0 \\
e \text { if } 0 \leq k<L \\
\top \text { if } k \geq L
\end{array}\right.
$$

Therefore, as $H_{1}^{\sharp}\left(z_{1}^{\prime}\right)$ is realizable, $H_{1}^{\sharp}\left(z_{1}^{\prime}\right) \succeq e_{1 L}$. Consequently, $z_{1}^{\prime} \succeq H_{1}\left(e_{1 L}\right) \oplus z_{1}$. Furthermore,

$$
H_{1}^{\sharp}\left(H_{1}\left(e_{1 L}\right) \oplus z_{1}\right) \succeq e_{1 L}
$$

Then, $H_{1}\left(e_{1 L}\right) \oplus z_{1}$ is feasible. Consequently, $z_{1}^{\prime}=$ $H_{1}\left(e_{1 L}\right) \oplus z_{1}$. In the next step, the least feasible output reference of the secondary subsystem $z_{2}^{\prime}$ greater than or equal to $z_{2}$ is calculated. By analogy with the main subsystem, $z_{2}^{\prime}=H_{2, z_{1}^{\prime}}\left(e_{2 L}\right) \oplus z_{2}$, where $e_{2 L}$ is a vector of appropriate dimension with all entries equal to $e_{L}$. In terms of complexity, the calculation of the least (with respect to $\preceq_{\Phi}$ ) feasible output reference greater than or equal to a predefined output reference $z$ is linear in $L$.

\subsection{Model Predictive Control}

A survey on MPC in classical control theory is available in Morari et al. (1989). MPC for max-plus linear systems has been widely studied (e.g., De Schutter and van den Boom (2001) and Necoara et al. (2008)). The principle of MPC for max-plus linear systems is briefly recalled in the following. At time $t$, the system response to an input is predicted using a model. Then, based on this output prediction, an optimal input is selected. At time $t+1$, the optimal input is applied to the system and the computation is repeated using event occurrences at time $t+1$. In the following, $K_{v, t}$ is defined as the index of the last occurrence of event $v$ before or at time $t$, i.e., at time $t$ event $v$ has occurred $K_{v, t}$ times.

For max-plus systems with partial synchronization, the aim is still to track the output reference $z$. To efficiently find the optimal input, the objective is reduced to a tracking horizon in terms of occurrences of events of size $M$. This leads to a specific output reference $z_{t}$ at time $t$ defined by

$$
\left(z_{t}\right)_{i}(k)=\left\{\begin{array}{l}
y_{i}(k) \text { if } k \leq K_{y_{i}, t} \\
z_{i}(k) \oplus 1 t \text { if } K_{y_{i}, t}<k \leq K_{y_{i}, t}+M \\
\top \text { if } k>K_{y_{i}, t}+M
\end{array}\right.
$$

with $1 \leq i \leq p$. $K_{u_{j}, t}+1$ occurrences of input event $u_{j}$ with $1 \leq j \leq m$ are sufficient to induce $K_{y_{i}, t}+1$ occurrences of output event $y_{i}$. Therefore, as a max-plus system with partial synchronization is event-invariant, $K_{u_{j}, t}+M+1$ occurrences of input event $u_{j}$ with $1 \leq j \leq m$ are sufficient to induce $K_{y_{i}, t}+M+1$ occurrences of output event $y_{i}$. Then, the optimal input computed at time $t$, denoted $u_{t}$, fulfills

$$
\left(u_{t}\right)_{j}(k)=\left\{\begin{array}{l}
u_{j}(k) \text { if } k \leq K_{u_{j}, t} \\
\top \text { if } k>K_{u_{j}, t}+M
\end{array}\right.
$$

with $1 \leq j \leq m$. Consequently, it remains to determine the occurring times of input event $\left(u_{t}\right)_{j}$ for occurrences $K_{u_{j}, t}+l$ with $1 \leq l \leq M$ (i.e., the next $M$ occurrences of input event $\left.\left(u_{t}\right)_{j}\right)$. A similar reasoning leads to the same results for state and output events. Then, to solve the control problem over a tracking horizon of size $M$, it is only necessary to predict the next $M$ occurrences of each event. Therefore, the tracking horizon corresponds to a prediction horizon. A fundamental difference between this approach and MPC in classical control theory is that the prediction horizon lies in the event domain while the iteration is done in the time domain. Therefore, at time $t$, the prediction horizon associated with an event may or may not shift depending on the occurrence of this event at time $t$. Consequently, the starting and ending occurrences of prediction horizons associated with different events might be different. A natural tuning parameter in this approach is the size $M$ of the tracking horizon. The method to select the optimal input comes down to calculate the optimal control while considering the history of the system. In Menguy et al. (2000), this problem is solved using operatorial representation. The idea of using residuation theory to solve the optimization problem in MPC is borrowed from Necoara et al. (2008).

As before, due to the priority of the main subsystem over the secondary subsystem, the control problem is first solved for the main subsystem by neglecting the secondary subsystem. The output reference of the main subsystem $z_{1 t}$ might not be feasible. Then, the output reference of the main subsystem is relaxed to ensure the existence of a realizable input. As in optimal control, the feasible reference output $z_{1 t}^{\prime}$ is defined by

where $u_{1 t}^{\prime}$ is defined by

$$
z_{1 t}^{\prime}=z_{1 t} \oplus H_{1}\left(u_{1 t}^{\prime}\right)
$$

$$
\left(u_{1 t}^{\prime}\right)_{j}(k)=\left\{\begin{array}{l}
\left(u_{1}\right)_{j}(k) \text { if } k \leq K_{\left(u_{1}\right)_{j}, t} \\
1 t \text { if } K_{\left(u_{1}\right)_{j}, t}<k \leq K_{\left(u_{1}\right)_{j}, t}+M \\
\top \text { if } k>K_{\left(u_{1}\right)_{j}, t}+M
\end{array}\right.
$$

with $1 \leq j \leq m_{1}$. The only terms which might differ between $\left(z_{1 t}\right)_{i}$ and $\left(z_{1 t}^{\prime}\right)_{i}$ are for occurrences $K_{\left(y_{1}\right)_{i}, t}+l$ with $1 \leq l \leq M$. These terms are calculated using (1). Second, the missing coefficients of $u_{1 t}$ are obtained with (4). To ensure linear performance in $M$, a componentwise approach is preferred to a matrix one: componentwise recursive equations are derived from (1) and (4). 
After predicting the state of the main subsystem using the computed optimal input, the optimal input of the secondary subsystem is obtained using a similar method. Due to the implicit equation in (2), it is necessary to use, in the prediction, a particular order for the events depending on the structure of the system (see David-Henriet et al. (2013)). These steps are also linear in $M$. Therefore, in terms of complexity, the optimization step for MPC with a tracking horizon of size $M$ is linear in $M$.

Example \%. A simple max-plus system with partial synchronization is considered. The main subsystem is described by

$$
\left\{\begin{array}{l}
x_{1}(k)=\left(\begin{array}{ll}
\varepsilon & e \\
\varepsilon & 2
\end{array}\right) x_{1}(k-1) \oplus\left(\begin{array}{l}
e \\
2
\end{array}\right) u_{1}(k) \\
y_{1}(k)=\left(\begin{array}{ll}
\varepsilon & e
\end{array}\right) x_{1}(k)
\end{array}\right.
$$

The secondary subsystem is described by

$$
\left\{\begin{aligned}
& x_{2}(k) \succeq\left(\begin{array}{ll}
\varepsilon & \varepsilon \\
1 & \varepsilon
\end{array}\right) x_{2}(k) \oplus\left(\begin{array}{ll}
\varepsilon & e \\
\varepsilon & \varepsilon
\end{array}\right) x_{2}(k-1) \\
& \oplus\left(\begin{array}{l}
e \\
\varepsilon
\end{array}\right) u_{2}(k) \\
& y_{2}(k)=\left(\begin{array}{ll}
\varepsilon & e
\end{array}\right) x_{2}(k) \\
& \operatorname{lm}\left(\left(x_{2}\right)_{1}\right) \subseteq \Phi_{1}
\end{aligned}\right.
$$

where $\left(x_{2}\right)_{1}$ is partially synchronized by $\left(x_{1}\right)_{2}$. The output references for the main subsystem and the secondary subsystem are

$$
\begin{aligned}
& \left(z_{1}(k)\right)_{0 \leq k \leq 8}=\{3,5,6,12,15,16,19,22, \top\} \\
& \left(z_{2}(k)\right)_{0 \leq k \leq 4}=\{4,8,15,21, \top\}
\end{aligned}
$$

Furthermore, a perturbation is assumed: occurrence 6 of $\left(x_{1}\right)_{1}$ occurs at the earliest at time $t=20$. For a tracking horizon of size 2 , the following results are obtained:

$$
\begin{aligned}
& \left(u_{1}(k)\right)_{0 \leq k \leq 8}=\{1,3,5,10,13,15,17,22, \top\} \\
& \left(y_{1}(k)\right)_{0 \leq k \leq 8}=\{3,5,7,12,15,17,22,24, \top\} \\
& \left(u_{2}(k)\right)_{0 \leq k \leq 4}=\{3,5,7,21, \top\} \\
& \left(y_{2}(k)\right)_{0 \leq k \leq 4}=\{4,6,8,23, \top\}
\end{aligned}
$$

MPC takes into account the perturbation on $\left(x_{1}\right)_{1}$ by delaying occurrence 7 of $u_{1}$ and occurrence 3 of $u_{2}$. For a tracking horizon of size 3 , the following results are obtained:

$$
\begin{aligned}
& \left(u_{1}(k)\right)_{0 \leq k \leq 8}=\{0,2,4,10,12,14,17,22, \top\} \\
& \left(y_{1}(k)\right)_{0 \leq k \leq 8}=\{2,4,6,12,14,16,22,24, \top\} \\
& \left(u_{2}(k)\right)_{0 \leq k \leq 4}=\{2,4,14,22, \top\} \\
& \left(y_{2}(k)\right)_{0 \leq k \leq 4}=\{3,5,15,23, \top\}
\end{aligned}
$$

With a tracking horizon of size 3 , the output reference of the main subsystem is respected. In this case, the performance is better with a tracking horizon of size 3 than with a tracking horizon of size 2 .

\section{CONCLUSION}

In this paper, the modeling of max-plus systems with partial synchronization has been investigated. A method to calculate the response to an input composed of daters over a finite horizon is presented. The control of max-plus systems with partial synchronization has also been studied. In the chosen approach, the performance of the main subsystem is not degraded to improve the performance of the secondary subsystem. This divides the control problem in two subproblems. First, the control problem is solved for the main subsystem by neglecting the secondary subsystem. Second, under a predefined behavior of the main subsystem, the control problem is solved for the secondary subsystem. This is expressed by optimality with respect to the lexico-graphic order $\preceq_{\Phi}$. The two considered control methods are optimal feedforward control and MPC. The main advantages of MPC over optimal feedforward control is the ability to react to perturbations and to update the output reference during run-time. Its main disadvantage is that calculations, linear in the size of the tracking horizon, are necessary during run-time to compute the next occurring times of input events. A major drawback for MPC applied to max-plus systems with partial synchronization in comparison to MPC applied to max-plus linear systems is that, at present, we cannot take constraints into account. A possible improvement is to use operatorial representation to develop MPC over an infinite tracking horizon.

\section{REFERENCES}

Baccelli, F., Cohen, G., Olsder, G.J., and Quadrat, J.P. (1992). Synchronization and Linearity, An Algebra for Discrete Event Systems. John Wiley and Sons, New York, USA.

Blyth, T.S. (2005). Lattices and Ordered Algebraic Structures. Springer, 1 edition.

Cohen, G., Gaubert, S., and Quadrat, J.P. (1993). From First to Second-Order Theory of Linear Discrete Event Systems. In 12th IFAC World Congress. Sydney.

Cohen, G., Moller, P., Quadrat, J.P., and Viot, M. (1989). Algebraic Tools for the Performance Evaluation of Discrete Event Systems. Proceedings of the IEEE, 77(1), 39-58. Special issue on Discrete Event Systems.

Cottenceau, B., Hardouin, L., Boimond, J.L., and Ferrier, J.L. (2001). Model Reference Control for Timed Event Graphs in Dioids. Automatica, 37(9), 1451-1458.

David-Henriet, X., Hardouin, L., Raisch, J., and Cottenceau, B. (2013). Optimal Control for Timed Event Graphs under Partial Synchronization. In $C D C^{\prime} 13$. Firenze, Italy.

De Schutter, B. and van den Boom, T.J.J. (2001). Model Predictive Control for Max-Plus-Linear Discrete Event Systems. Automatica, 37(7), 1049-1056.

De Schutter, B. and van den Boom, T.J.J. (2003). MPC for Discrete-Event Systems with Soft and Hard Synchronisation Constraints. International Journal of Control, $76(1), 82-94$.

Lhommeau, M., Hardouin, L., and Cottenceau, B. (2003). Optimal Control for $(\max ,+)$-linear Systems in the Presence of Disturbances. Positive Systems: Theory and Applications, POSTA, Springer LNCIS 294, 47-54.

Menguy, E., Boimond, J.L., Hardouin, L., and Ferrier, J.L. (2000). Just-in-Time Control of Timed Event Graphs: Update of Reference Input, Presence of Uncontrollable Input. IEEE Transactions on Automatic Control, 45(11), 2155-2159.

Morari, M., Garcia, C.E., and Prett, D.M. (1989). Model Predictive Control: Theory and Practice - A Survey. Automatica, 25(3), 335-348.

Necoara, I., van den Boom, T.J.J., De Schutter, B., and Hellendoorn, H. (2008). Stabilization of Max-PlusLinear Systems Using Model Predictive Control: The Unconstrained Case. Automatica, 44(4), 971-981. 